\title{
Optical implementation of quantum random walks using weak cross-Kerr media
}

\author{
WANG Chuan ${ }^{1,2,3^{*}}$, LI YanSong ${ }^{3} \&$ HAO Liang ${ }^{3}$ \\ ${ }^{1}$ School of Science, Beijing University of Posts and Telecommunications, Beijing 100876, China; \\ ${ }^{2}$ Key Laboratory of Optical Communication and Lightwave Technologies, Beijing University of Posts and Telecommunications, Beijing 100876, China; \\ ${ }^{3}$ Department of Physics, Tsinghua University, Beijing 100084, China
}

Received January 23, 2011; accepted March 9, 2011

\begin{abstract}
Weak cross-Kerr media provides additional degrees of freedom of qubits in quantum information processing. In this paper, by exploiting weak cross-Kerr nonlinearity, we propose an optical implementation scheme of one-dimensional quantum random walks. The random walks are described by the interaction of single photons with cross-Kerr media. The proposed scheme can also be used to implement one-dimensional quantum random walks on an infinite line.
\end{abstract}

quantum random walks, cross-Kerr media

Citation: Wang C, Li Y S, Hao L. Optical implementation of quantum random walks using weak cross-Kerr media. Chinese Sci Bull, 2011, 56: 2088-2091, doi: $10.1007 / \mathrm{s} 11434-011-4545-5$

Quantum computers, which are based on the principles of quantum mechanics, provide a speedup of classical computers. Quantum algorithms are the key ingredients of quantum computation for specific problems. In 1994, Shor proposed the quantum factoring algorithm, which challenges the security of classical encryption systems [1]. The proposed algorithm can factorize a large number with two prime numbers within a finite time. Later in 1997, Grover constructed the quantum search algorithm that searches for a marked item with very high probability from an unsorted database with $N$ items using the Grover algorithm with only $O(\sqrt{N})$ steps [2]. It has been shown that under phase matching [3-5], one can find a marked state with certainty [6]. Much progress was made in quantum algorithms and quantum computation in the following decade [7-14]. However, it is difficult to find new quantum algorithms for quantum information processing.

Classical random walks are well known to solve NPcomplete problems. In the process of classical random walking, a particle moves along a two-way lattice. At each step, the particle moves to the left or to the right on the basis of the result of a coin flip. Quantum random walks are the quan-

*Corresponding author (email: wangchuan82@gmail.com) tum version of classical random walks, in which the classical coin is replaced with a quantum coin, which is used to extend the set of quantum algorithms. There are two different types of quantum random walks: discrete and continuous quantum random walks. In 1993, Aharonov et al. first proposed the idea of quantum walks that require an additional quantum coin to allow discrete unitary evolution [15]. Later, Farhi and Gutmann proposed another quantum random walk algorithm based on continuous unitary evolution [16]. Shenvi et al. demonstrated that a search algorithm based on quantum walks can achieve speeds as high as that of Grover's algorithm [17]. In the following decades, quantum random walks attracted a variety of interest and have been a topic of research in the field of quantum information processing. Methods for possible implementations of one dimensional quantum random walks have been suggested by different groups in various physical systems. In 2002, Travaglione et al. proposed the method of quantum random walks using ion traps [18]. Zähringer et al. experimentally realized a quantum walk with trapped ions on a line in phase space [19]. Later, Dur et al. proposed that quantum random walks can be realized using neutral atoms in optical lattices [20]. Zou et al. proposed the optical implementation of one dimensional two- 
state and three-state quantum random walks using single photons exploiting orbital angular momentum [21]. Their proposed scheme exploits linear optical devices and holograms that can be extended to an unlimited number steps in principle. Recently, Schreiber et al. proposed the implementation of a quantum walk using passive linear optical elements [22]. There was much related research on quantum random walks in the following years [23-25].

Recently, there has been considerable interest in quantum information processing based on weak cross-Kerr nonlinearities. As a quantum nondemolition measurement can be realized using the weak Kerr interaction, increasing efforts have been devoted to using cross-Kerr media in various protocols of quantum information processing, such as quantum gates [26-28], entanglement generation [29], entanglement purification and concentration [30, 31], and Bell state analysis [32]. In the above discussions, cross-Kerr media are used to denote the spatial information of the photons in the quantum nondemolition measurement and parity check. The cross-Kerr interaction of the single photon and coherent state system can be described by

$$
\begin{aligned}
U_{c k}(a|0\rangle+b|1\rangle)|\alpha\rangle & =e^{i \chi n_{a} n_{c}}(a|0\rangle+b|1\rangle)|\alpha\rangle \\
& =a|0\rangle|\alpha\rangle+b|1\rangle\left|\alpha e^{i \theta}\right\rangle,
\end{aligned}
$$

where $n_{a}\left(n_{c}\right)$ denotes the number operator for mode a (c) and $\chi$ is the coupling strength of the nonlinearity, and $|0\rangle$ and $|1\rangle$ represent the vacuum state and single-photon state, respectively. One can easily distinguish the spatial mode of the single photon or the numbers of photons by measuring the phase shift of coherent states without destroying the photons.

\section{Quantum random walk using cross-Kerr nonlinearity}

In the following, we briefly review the procedures of onedimensional quantum random walks for a single particle prepared in the state $|l\rangle$ that can move between discrete sites labeled $l$. The movement of the particle is determined by the state of a quantum coin. Here the state of the quantum coin is represented by $|u\rangle$ and $|d\rangle$ in two dimensional Hilbert space. Each step of the one-dimensional quantum random walk consists of two processes.

(i) A Hadamard operation is performed on the coin state, which is described as

$$
H=\left(\begin{array}{cc}
1 & 1 \\
1 & -1
\end{array}\right)
$$

and the coin state undergoes a Hadamard transformation, which is described by

$$
H\left(\begin{array}{l}
|u\rangle \\
|d\rangle
\end{array}\right)=\left(\begin{array}{cc}
1 & 1 \\
1 & -1
\end{array}\right)\left(\begin{array}{c}
|u\rangle \\
|d\rangle
\end{array}\right)=\left(\begin{array}{c}
|u\rangle+|d\rangle \\
|u\rangle-|d\rangle
\end{array}\right),
$$

where the position degree of freedom is denoted by the coin states labeled $|u\rangle$ and $|d\rangle$.

(ii) The state of the position undergoes a shift operation; here the movement of the position to the left or right is denoted by the operation $K_{1}$ :

$$
K_{1}|u\rangle|l\rangle=|u\rangle|l+1\rangle, \quad K_{1}|d\rangle|l\rangle=|d\rangle|l-1\rangle .
$$

After one step of the random walk, the state of the composite system can be described by

$$
\begin{aligned}
& |u\rangle|l\rangle \rightarrow \frac{1}{\sqrt{2}}(|u\rangle|l+1\rangle+|d\rangle|l-1\rangle), \\
& |d\rangle|l\rangle \rightarrow \frac{1}{\sqrt{2}}(|u\rangle|l+1\rangle-|d\rangle|l-1\rangle) .
\end{aligned}
$$

By iteration of the random walks, the one-dimensional quantum random walk can be implemented with unlimited steps.

Here we exploit the spatial mode of single photons as the coin states spanning the two-dimensional Hilbert space, which means that the coin states can be described by the two spatial modes of the single photon, say the upper mode $|u\rangle$ and lower mode $|d\rangle$. The two spatial modes become superposed by the operation of beamsplitters as the states $\frac{1}{\sqrt{2}}(|u\rangle+|d\rangle)$ and $\frac{1}{\sqrt{2}}(|u\rangle-|d\rangle)$ of the two output modes. The principles of the experimental setup are shown in Figure 1. The analysis of quantum random walks using Kerr media is described in detail as follows. When the algorithm of the random walk starts, we assume that the initial state is prepared in $|d\rangle|\alpha\rangle$. This indicates one single photon pulse is produced and projected from the lower mode of input port $I_{2}$ and a coherent beam in the state $|\alpha\rangle$ is generated using lasers and passes through the input port $I_{1}$.

In the first step of the random walk, the $K_{1}$ operation is realized by cross-Kerr interaction between the single photon and coherent state. The evolution of the operation is described as

$$
K_{1}|u\rangle|\alpha\rangle=|u\rangle\left|\alpha e^{i \theta}\right\rangle, \quad K_{1}|d\rangle|\alpha\rangle=|d\rangle\left|\alpha e^{-i \theta}\right\rangle,
$$

where we denote the cross-Kerr interaction as the operation $K_{1}$. As shown in Figure 1, it is obvious that each step of the quantum random walk consists of two operations, say a Hadamard operation and shift operation. The coin state

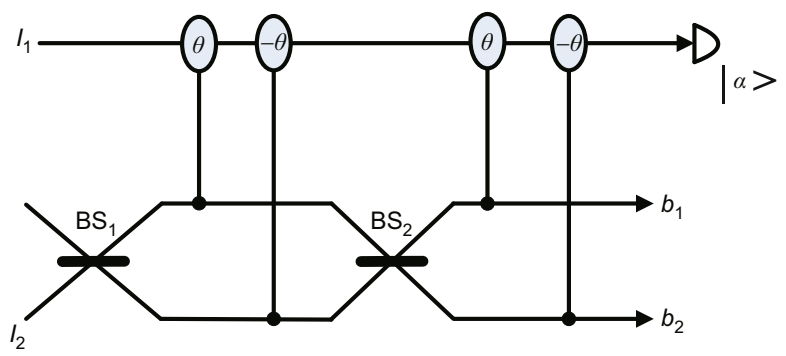

Figure 1 Schematic diagram showing the principle of one-dimensional quantum random walks. Cross-Kerr media are used in the setup. $\theta$ denotes the phase shift introduced by cross-Kerr media. BS represents a 50:50 beam splitter. $I_{1}$ and $I_{2}$ represent the coherent state input and single photon input, respectively. 
undergoes the Hadamard transformation and the shift operation can be described as

$$
\begin{aligned}
& |u\rangle|\alpha\rangle \longrightarrow \frac{1}{\sqrt{2}}\left(|u\rangle\left|\alpha e^{i \theta}\right\rangle+|d\rangle\left|\alpha e^{-i \theta}\right\rangle\right), \\
& |d\rangle|\alpha\rangle \longrightarrow \frac{1}{\sqrt{2}}\left(|u\rangle\left|\alpha e^{i \theta}\right\rangle-|d\rangle\left|\alpha e^{-i \theta}\right\rangle\right),
\end{aligned}
$$

where the phases in the coherent state are used to mark the positions of the particle. After one step of the random walk, the distribution probabilities are $50 \%$ at both $\theta$ and $-\theta$ positions.

Suppose that the initial state is prepared in $|d\rangle|\alpha\rangle$. After interaction with the cross-Kerr media as shown in Figure 1, the state evolves as

$$
|d\rangle|\alpha\rangle \longrightarrow \frac{1}{\sqrt{2}}\left(|u\rangle\left|\alpha e^{i \theta}\right\rangle-|d\rangle\left|\alpha e^{-i \theta}\right\rangle\right),
$$

where the phase factors $\theta$ and $-\theta$ represent the positions of the particle in the random walk process. After the second iteration of the two steps, the evolution of the composite system is described by

$$
\begin{aligned}
& \frac{1}{\sqrt{2}}\left(|u\rangle\left|\alpha e^{i \theta}\right\rangle-|d\rangle\left|\alpha e^{-i \theta}\right\rangle\right) \longrightarrow \\
& \frac{1}{2}\left(|u\rangle\left|\alpha e^{2 i \theta}\right\rangle+|d\rangle|\alpha\rangle-|u\rangle|\alpha\rangle+|d\rangle\left|\alpha e^{-2 i \theta}\right\rangle\right) .
\end{aligned}
$$

After two steps of the random walk, the distribution probabilities are similar to those of the classical random walk. The distribution probabilities are $25 \%$ at position $+2 \theta$ and position $-2 \theta$ and $50 \%$ at position 0 . We then perform the next step of the quantum random walk, and the system evolves as

$$
\begin{aligned}
& \frac{1}{2}\left(|u\rangle\left|\alpha e^{2 i \theta}\right\rangle+|d\rangle|\alpha\rangle-|u\rangle|\alpha\rangle+|d\rangle\left|\alpha e^{-2 i \theta}\right\rangle\right) \\
\longrightarrow \quad & \frac{1}{2 \sqrt{2}}\left(|u\rangle\left|\alpha e^{3 i \theta}\right\rangle-2|u\rangle\left|\alpha e^{i \theta}\right\rangle-|d\rangle\left|\alpha e^{i \theta}\right\rangle\right. \\
& \left.+|u\rangle\left|\alpha e^{-i \theta}\right\rangle-|d\rangle\left|\alpha e^{-3 i \theta}\right\rangle\right) \\
& =\frac{1}{2 \sqrt{2}}\left[|u\rangle\left|\alpha e^{3 i \theta}\right\rangle-(2|u\rangle+|d\rangle)\left|\alpha e^{i \theta}\right\rangle\right. \\
& \left.+|u\rangle\left|\alpha e^{-i \theta}\right\rangle-|d\rangle\left|\alpha e^{-3 i \theta}\right\rangle\right] .
\end{aligned}
$$

The probability of the distribution is $5 / 8$ at position $+\theta$, and it is obvious that the probability distribution differs from that for classical random walks. By iteration of the above steps, quantum random walks can be realized in our proposed setup. As the position of a particle is represented by the phase degree of freedom, the optical network shown in Figure 2 indicates that one-dimensional quantum random walks can be realized with arbitrary steps.

The probability of distribution in the quantum case differs from that in the classical case and depends on the initial state of the random walks. Quantum random walks result in an asymmetric distribution of position, say drift to the left or to the right. The symmetric condition can be achieved if the initial state is prepared as the superposed state $\frac{1}{\sqrt{2}}(|u\rangle+|d\rangle)$.

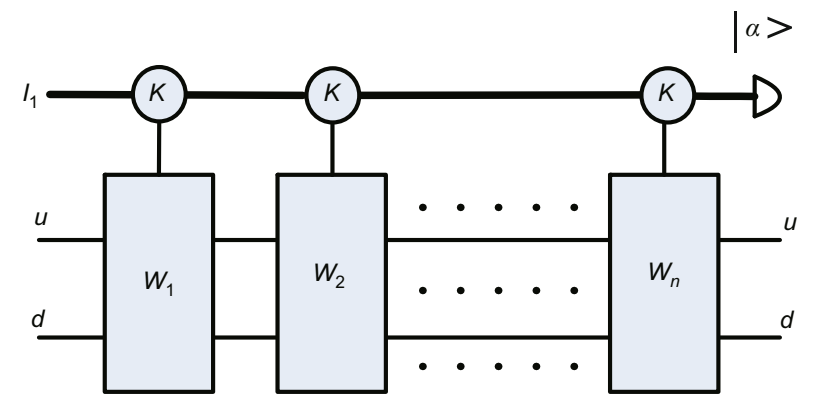

Figure 2 Construction of an infinite quantum random walk network using cross-Kerr media. Each $K$ in the figure represents one step of the quantum coin toss.

\section{Experimental feasibility}

In this study, we proposed the implementation of quantum random walks using cross-Kerr media and homodyne detection. However, the implementation of the cross-Kerr effect with single photons can be readily affected by noise [33, 34], and the efficiency of homodyne detection may be not perfect under realistic conditions. For example, single-photon crossKerr nonlinearity is a controversial assumption with current technology. As discussed in [34], a large phase shift via a giant Kerr effect in the case of single-photon wave packets is impossible. However, there has been much discussion on establishing a feasible scheme by choosing suitable Kerr nonlinear media and good measurement strategies for the coherent state [35].

In the present scheme, homodyne detections are made to distinguish the phase shift of coherent states. The problem with our proposed scheme is that detecting phase shift is not easy, which means that the phase shifts $\theta$ and $\theta^{\prime}$ of the coherent states are relatively small [26]. As the distribution functions in phase spaces of a coherent state can be described by $f(x, \alpha \cos \theta)=\exp \left[-(x-2 \alpha \cos \theta)^{2} / 4\right] /(2 \pi)^{1 / 4}$, the distribution functions are Gaussian curves. Suppose that the particle undergoes a three-step random walk and the evolution of the composite system is obtained:

$$
\begin{array}{r}
\frac{1}{2 \sqrt{2}}\left[|u\rangle\left|\alpha e^{3 i \theta}\right\rangle-(2|u\rangle+|d\rangle)\left|\alpha e^{i \theta}\right\rangle\right. \\
\left.+|u\rangle\left|\alpha e^{-i \theta}\right\rangle-|d\rangle\left|\alpha e^{-3 i \theta}\right\rangle\right] .
\end{array}
$$

Using the expression $\langle X \mid \alpha\rangle$, we have $f(x, \alpha \cos 3 \theta)|u\rangle-$ $f(x, \alpha \cos \theta)(2|u\rangle+|d\rangle)+f(x,-\alpha \cos \theta)|u\rangle-f(x,-\alpha \cos 3 \theta)|d\rangle$ after the measurement. The key point of the protocol is to distinguish the phases of the coherent state which means that $f(x, \alpha \cos 3 \theta), f(x, \alpha \cos \theta), f(x,-\alpha \cos 3 \theta)$ and $f(x,-\alpha \cos \theta)$ must be readout deterministically. As these expressions are Gaussian curves in phase space and the peaks are separated by a distance $X_{d}=\alpha_{1}-\alpha_{2}$, here $\alpha_{1}, \alpha_{2} \in$ $\{\alpha \cos 3 \theta,-\alpha \cos 3 \theta, \alpha \cos \theta,-\alpha \cos \theta\}$. As discussed in [36], the nonlinearities can reach $\theta \sim 10^{-2}$ employing electromagnetically induced transparency. Therefore, the phases $\theta$ and 
$\theta^{\prime}$ are not easy to be distinguished by direct homodyne measurement. Therefore, the detection may introduce errors in our scheme. The probability of a discrimination error occurring is represented by a complementary error function:

$$
\frac{1}{2} \operatorname{Erfc}\left[X_{d} / 2 \sqrt{2}\right]
$$

\section{Summary}

Exploiting weak cross-Kerr nonlinearities, one-dimensional quantum random walks are realized using current optical technologies. Our proposed scheme only requires linear optical elements and homodyne detectors. The initial state consists of a single photon and coherent state. The spatial modes of the single photon represent the quantum coin state and are operated by on-line beamsplitters. Through cross-Kerr interaction, the information of spatial modes can be transmitted to phase information of the coherent state. One can mark the positions of a random walk by reading out the phases of the coherent state. Additionally, the implementation can be generalized in principle to an infinite line by iterating the same procedures with unlimited steps.

This work was supported by the National Basic Research Program of China (2010CB923202), the Specialized Research Fund for Doctoral Programs of the Ministry of Education of China (20090005120008), the Fundamental Research Funds for the Central Universities (BUPT2009RC0710) and the National Natural Science Foundation of China (10805010, 10947151).

1 Shor P. Algorithms for quantum computation: Discrete logarithms and factoring. In: Proceedings of 35th Annual Symposium on Foundations of Computer Science, 1994, IEEE Press, Los Alamitos, CA

2 Grover L. Quantum mechanics helps in searching for a needle in a haystack. Phys Rev Lett, 1997, 79: 325-328

3 Long G L, Li Y S, Zhang W L, et al. Phase matching in quantum searching. Phys Lett A, 1999, 262: 27-34

4 Long G L, Xiao L, Sun Y. Phase matching condition for quantum search with a generalized initial state. Phys Lett A, 2002, 294: 143-152

5 Bhattacharya $\mathrm{N}$, van Linden $\mathrm{H} \mathrm{B}$, van den $\mathrm{H}$, et al. Implementation of quantum search algorithm using classical fourier optics. Phys Rev Lett, 2002, 88: 137901

6 Long G L. Grover algorithm with zero theoretical failure rate. Phys Rev A, 2001, 64: 022307

7 Wang A M. Quantum central processing unit and quantum algorithm. Chin Phys Lett, 2002, 19: 620-622

8 Ding S C, Jin Z. Review on the study of entanglement in quantum computation speedup. Chinese Sci Bull, 2007, 52: 2161-2166

9 Yang X D, Wei D X, Luo J, et al. Preparation of pseudopure state in nuclear spin ensemble using CNOT gates combination. Chinese Sci Bull, 2002, 47: 1856-1860

10 Hao L, Liu D, Long G L. An N/4 fixed-point duality quantum search algorithm. Sci China Ser G-Phys Mech Astron, 2010, 53: 1765-1768
11 Zhang J F, Xie J Y, Deng Z W, et al. Dense coding scheme using superpositions of Bell-states and its NMR implementation. Sci China Ser G-Phys Mech Astron, 2005, 48: 57-67

12 Liu W Z, Zhang J F, Deng Z W, et al. Simulation of general three-body interactions in a nuclear magnetic resonance ensemble quantum computer. Sci China Ser G-Phys Mech Astron, 2008, 51: 1089-1096

13 Long G L. General quantum interference principle and duality computer. Commun Theor Phys, 2006, 45: 825-844

14 Long G L, Liu Y. Duality computing in quantum computers. Commun Theor Phys, 2008, 50: 1303-1306

15 Aharonov Y, Davidovich L, Zagury N. Quantum random walks. Phys Rev A, 1993, 48: 1687-1690

16 Farhi E, Gutmann S. Quantum computation and decision trees. Phys Rev A, 1998, 58: 915-928

17 Shenvi N, Kempe J, Whaley K B. Quantum random-walk search algorithm. Phys Rev A, 2003, 67: 052307

18 Travaglione B C, Milburn G J. Implementing the quantum random walk. Phys Rev A, 2002, 65: 032310

19 Zähringer F, Kirchmair G, Gerritsma R, et al. Realization of a quantum walk with one and two trapped ions. Phys Rev Lett, 2010, 104: 100503

20 Dur W, Rausendorf R, Kendon V M, et al. Quantum walks in optical lattices. Phys Rev A, 2002, 66: 052319

21 Zou X B, Dong Y L, Guo G C. Optical implementation of onedimensional quantum random walks using orbital angular momentum of a single photon. New J Phys, 2006, 8: 81

22 Schreiber A, Cassemiro K N, Potocek V, et al. Photons walking the line: a quantum walk with adjustable coin operations. Phys Rev Lett, 2010 , 104: 050502

23 Knight P L, Roldan E, Sipe J E. Quantum walk on the line as an interference phenomenon. Phys Rev A, 2003, 68: 020301

24 Jeong H, Paternostro M, Kim M S. Simulation of quantum random walks using the interference of a classical field. Phys Rev A, 2004 , 69: 012310

$25 \mathrm{Ma} \mathrm{L}, \mathrm{Du} \mathrm{J}$ F, Li Y, et al. White noise in quantum random walk search algorithm. Chin Phys Lett, 2006, 23: 779-782

26 Nemoto K, Munro W J. Nearly deterministic linear optical controlledNOT gate. Phys Rev Lett, 2004, 93: 250502

27 Lin Q, He B. Single-photon logic gates using minimal resources. Phys Rev A, 2009, 80: 042310

28 He B, Ren Y, Bergou J A. Creation of high-quality long-distance entanglement with flexible resources. Phys Rev A, 2009, 79: 052323

29 Jin G S, Lin Y, Wu B. Generating multiphoton Greenberger-HorneZeilinger states with weak cross-Kerr nonlinearity. Phys Rev A, 2007, 75: 054302

30 Sheng Y B, Deng F G, Zhou H Y. Efficient polarization-entanglement purification based on parametric down-conversion sources with crossKerr nonlinearity. Phys Rev A, 2008, 77: 042308

31 Sheng Y B, Deng F G, Zhou H Y. Nonlocal entanglement concentration scheme for partially entangled multipartite systems with nonlinear optics. Phys Rev A, 2008, 77: 062325

32 Barrett S D, Kok P, Nemoto K, et al. Symmetry analyzer for nondestructive Bell-state detection using weak nonlinearities. Phys Rev A, 2005, 71: 060302(R)

33 Shapiro J H. Single-photon Kerr nonlinearities do not help quantum computation. Phys Rev A, 2006, 73: 062305

34 Gea-Banacloche J. Impossibility of large phase shifts via the giant Kerr effect with single-photon wave packets. Phys Rev A, 2010, 81: 043823

35 Pryde G J, O'Brien J L, White A G, et al. Measuring a photonic qubit without destroying it. Phys Rev Lett, 2004, 92: 190402

36 Munro W J, Nemoto K, Beausoleil R G, et al. High-efficiency quantumnondemolition single-photon-number-resolving detector. Phys Rev A, 2005, 71: 033819

Open Access This article is distributed under the terms of the Creative Commons Attribution License which permits any use, distribution, and reproduction in any medium, provided the original author(s) and source are credited. 